\section{Reducing Disparities in Cervical Cancer Mortality Among Young Black Women}

\author{
Nadeem R. Abu-Rustum, MD
}

A Ithough the overall incidence of cervical cancer between Black and White women is now similar, the retrospective cohort study by Alimena et al ${ }^{1}$ published in this issue highlights how young Black women (age $\leq 39$ years) remain particularly vulnerable to presenting with more advanced cervical cancers and having worse overall survival outcomes. The authors suggest that this finding may be related to insurance-related disparities and inadequate follow-up for abnormal Papanicolaou (Pap) test results.

There are several other reasons for these health inequities among young black women, as well as proposed methods for overcoming them. These measures include building stronger trust between young Black women and gynecologic healthcare providers and systems; increased HPV vaccination acceptance and delivery; increased Pap test screening availability and outreach screening in nontraditional sites ${ }^{2}$; prompt follow-up for abnormal Pap test results; prompt treatment for severe precancerous abnormalities; providing basic necessary resources for curative treatment, including access to gynecologic oncology and radiation oncology specialists, when cancer is detected ${ }^{3,4}$; transportation assistance; implementation of socially determined cervical cancer care navigation programs at public safety-net hospitals to improve treatment adherence ${ }^{5}$; and access to financial assistance programs to help cover the cost of treatment when medical insurance is not available. The development of dedicated public centers in every state is needed for the establishment of healthcare processes that provide this kind of quality care to young, Black, uninsured or underinsured women.

Encouraging results from the study by Alimena et al ${ }^{1}$ include the diminishing disparities with increasing age among Black women, with no reported disparity in Black women aged $\geq 65$ years, and more importantly, the absence of disparities when optimal treatment for cervical cancer is delivered. Receiving optimal, guideline-directed treatment has to do with access to quality care, trust of healthcare systems, and the acceptance/compliance of patients to receive treatment recommendations that may be time-consuming and require time away from family and other personal responsibilities. ${ }^{6}$ Family and social support are always important during cancer treatment, as treatment modalities such as surgery and chemoradiation are usually very demanding on patients.

In summary, having access to gynecologic oncologists and radiation oncologists and complying with treatment recommendations eliminates discrepancies of age and race in relation to the management of cervical cancer. ${ }^{7}$ The good news is the problem does not appear to be related to unique histology or biological differences specific to Black women, and the cited barriers can be addressed and overcome with continued efforts to improve trust of gynecologic healthcare systems and increasing access and compliance with treatment. I applaud the authors in their efforts to bring more awareness to this timely and important subject.

See page 789 for related article.

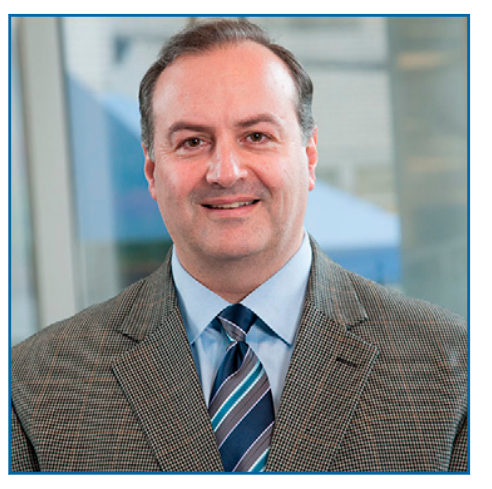

NADEEM R. ABU-RUSTUM, MD

Nadeem R. Abu-Rustum, MD, is Chief of the Gynecologic Oncology Service in the Department of Surgery at Memorial Sloan Kettering Cancer Center (MSKCC), where he also serves as Vice-Chair for Technology Development. Additionally, he is the Avon Chair in Gynecologic Oncology and Professor of Obstetrics and Gynecology at Weill Cornell Medical College.

Dr. Abu-Rustum received his medical degree from the American University of Beirut in Lebanon. He completed his residency in obstetrics and gynecology at the Greater Baltimore Medical Center and a fellowship in gynecologic oncology at MSKCC.

Dr. Abu-Rustum's clinical research focuses on surgical therapy for gynecologic cancers and innovative surgical approaches, including sentinel node mapping, improved localization and visualization of tumors, fertility-sparing surgery, and laparoscopy. He also has extensive experience in endometrial and cervical cancer and fertility-sparing radical trachelectomy and has numerous contributions to the field of gynecologic cancer surgery, for which he received the Society of Gynecologic Oncology SGO Innovation Award in 2017 for his work on sentinel node mapping of uterine cancer.

In his current role at MSKCC, he is actively involved in the development and improvement of surgical techniques and oversees surgical research programs. Over the years, he has built collaborations with the gynecologic oncology team at the Medical University of Vienna and has been appointed adjunct Professor in the Department of Obstetrics and Gynecology at the Medical University of Vienna.

Dr. Abu-Rustum is Chair of the NCCN Cervical/ Uterine/Vulva/GTD Cancers Panels, and has served as a member of the Gynecologic Oncology Group Cervix Committee and the American College of Surgeons Committee on Emerging Surgical Technology and Education. He has authored or coauthored more than 250 publications and is coauthor to numerous book chapters and 3 textbooks in gynecologic oncology.

\section{doi: 10.6004/jnccn.2021.7069}

The ideas and viewpoints expressed in this commentary are those of the author and do not necessarily represent any policy, position, or program of NCCN. 
Disclosures: Dr. Abu-Rustum has disclosed receiving institutional grants from Stryker/Novadaq, Olympus, and GRAIL.
Correspondence: Nadeem R. Abu-Rustum, MD, Gynecology Service, Department of Surgery, Memorial Sloan Kettering Cancer Center, 1275 York Avenue, New York, NY 10065. Email: Abu-rusn@mskcc.org

\section{References}

1. Alimena S, Pachigolla SL, Feldman S, et al. Race- and age-related disparities in cervical cancer mortality. J Natl Compr Canc Netw 2021;19: 789-795.

2. Massad SL, Cejtin HE, Abu-Rustum NR. Presentation and screening history of indigent women with cervical cancer: implications for prevention. J Low Genit Tract Dis 2000;4:208-211.

3. Brookfield KF, Cheung MC, Lucci J, et al. Disparities in survival among women with invasive cervical cancer: a problem of access to care. Cancer 2009;115:166-178.

4. Ramondetta LM, Meyer LA, Schmeler KM, et al. Avoidable tragedies: disparities in healthcare access among medically underserved women diagnosed with cervical cancer. Gynecol Oncol 2015;139: 500-505.

5. Dessources K, Hari A, Pineda E, et al. Socially determined cervical cancer care navigation: an effective step toward health care equity and care optimization. Cancer 2020;126:5060-5068.

6. Abu-Rustum NR, Lee S, Correa A, Massad LS. Compliance with and acute hematologic toxic effects of chemoradiation in indigent women with cervical cancer. Gynecol Oncol 2001;81:88-91.

7. Behbakht K, Abu-Rustum NR, Lee $S$, et al. Characteristics and survival of cervical cancer patients managed at adjacent urban public and university medical centers. Gynecol Oncol 2001;81:40-46. 